doi:10.13108/2020-12-4-119

\title{
INVERSE PROBLEM FOR FRACTIONAL ORDER PSEUDO-PARABOLIC EQUATION WITH INVOLUTION
}

\author{
D. SERIKBAEV
}

\begin{abstract}
In this paper, we consider an inverse problem on recovering the right-hand side of a fractional pseudo-parabolic equation with an involution operator. The major obstacle for considering the inverse problems is related with the well-posedness of the problem. Inverse problems are often ill-posed. For example, the inverse heat equation, deducing a previous distribution of temperature from final data, is not well-posed since the solution is highly sensitive to variations in the final data.

The advantage of this paper is two-fold. On the one hand, we investigate the solvability of the direct problem and prove the solvability to this problem. On the other hand, we study the inverse problem based on this direct problem and prove the solvability results in this problem, too.

First, we investigate the Cauchy problem for the time-fractional pseudo-parabolic equation with the involution operator, and secondly, we consider the inverse problem on recovering the right-hand side from an overdetermined final condition and prove that it is solvable.

To achieve our goals, we use methods corresponding to the different areas of mathematics such as the theory of partial differential equations, mathematical physics, and functional analysis. In particular, we use the $\mathcal{L}$-Fourier analysis method to establish the existence and uniqueness of solutions to this problem on the Sobolev space.

The classical and generalized solutions of the inverse problem are studied.
\end{abstract}

Keywords: fractional differential equation, inverse problem, involution, pseudo-parabolic equation.

Mathematics Subject Classification 2010: 35R30, 34K37

\section{INTRODUCTION}

In this paper we study the inverse problem for the nonlocal pseudo-parabolic equation with an involution of the space variable $x$. We investigate the equation

$$
\mathcal{D}_{t}^{\alpha}\left[u(t, x)-u_{x x}(t, x)+\varepsilon u_{x x}(t, \pi-x)\right]-u_{x x}(t, x)+\varepsilon u_{x x}(t, \pi-x)=f(x),
$$

for

$$
(t, x) \in \Omega=\{0<t<T<\infty, 0<x<\pi\}, \quad 0<\alpha \leqslant 1,
$$

where $\mathcal{D}_{t}^{\alpha}$ is the Caputo derivative (see [14]) for $0<\alpha<1$ and $\mathcal{D}_{t}^{\alpha}:=\partial_{t}$ for $\alpha=1$. In $L^{2}(0, \pi)$ we consider a second order differential operator generated by the differential expression

$$
\mathcal{L}(u)=-u^{\prime \prime}(x)+\varepsilon u^{\prime \prime}(\pi-x), \quad 0<x<\pi,
$$

D. Serikbaev, Inverse Problem for fractional order PSEUdo-Parabolic Equation with invoLUTION.

(C) D. Serikbaev 2020.

The author was supported by the Science Committee of the Ministry of Education and Science of the Republic of Kazakhstan Grant AP08052425 and By the FWO Odysseus 1 grant G.0H94.18N: Analysis and Partial Differential Equations.

Submitted October 16, 2020. 
subject to the boundary conditions

$$
u(0)=0, \quad u(\pi)=0,
$$

where $|\varepsilon|<1, \varepsilon \in \mathbb{R}$. We can check easily that the introduced operator is self-adjoint (see [1, 13, 28]). For all $|\varepsilon|<1$, nonlocal problem (1.2), 1.3) has the following eigenvalues:

$$
\lambda_{2 k}=4(1+\varepsilon) k^{2}, k \in \mathbb{N} \quad \text { and } \quad \lambda_{2 k+1}=(1-\varepsilon)(2 k+1)^{2}, \quad k \in \mathbb{Z}_{+},
$$

and the corresponding system of eigenfunctions

$$
\begin{aligned}
& u_{2 k}(x)=\sqrt{\frac{2}{\pi}} \sin 2 k x, \quad k \in \mathbb{N}, \\
& u_{2 k+1}(x)=\sqrt{\frac{2}{\pi}} \sin (2 k+1) x, \quad k \in \mathbb{N} \cup\{0\} .
\end{aligned}
$$

As a motivation, we mention that pseudo-parabolic equations have numerous applications in sciences and engineering. For example, the energy functions of the isotropic materials can be expressed as solutions of pseudo-parabolic equations [5]. Some wave processes [3], filtration of the two-phase flow in porous media with the dynamic capillary pressure [4] are also modeled by pseudo-parabolic equations. The time-fractional pseudo-parabolic equation (1.1) occurs in studying flows of the Oldroyd-B fluid, one of the most important classes of dilute solutions of polymers 7,27 .

The study of inverse problems for pseudo-parabolic equations was initiated in the 1980s. The first result obtained by Rundell [22] refers to the inverse identification problems for an unknown source function $f$ in the following equation

$$
\frac{\partial}{\partial t}[u(t, x)+L u(t, x)]+L u(t, x)=f,
$$

where $L$ is an even order linear differential operator. Rundell proved global existence and uniqueness theorems for then cases when $f$ depends either only on $x$ or only on $t$.

The inverse problems on identifying the right hand sides of the pseudo-parabolic equations from a local over-determination condition have important applications in various areas of applied sciences and engineering. Inverse source problems for the diffusion, sub-diffusion and for other types of equations are well studied. In this area some recent progresses have been done in the series of articles, see, for example, $1,2,2,9,10,12,13,15,21,23,24,26,28$. However, inverse problems for pseudo-parabolic equations and for their fractional analogues have been studied relatively less, see $8,11,17,19,22$. In our paper, we aim to fill this gap.

For more information on pseudo-parabolic equations, we refer to a book by Demidenko and Uspenskii [6] and the references therein.

\section{DireCt PROBLEM}

Before formulating a problem, we first introduce fractional differentiation operators.

Definition 2.1. The Riemann-Liouville fractional integral $I^{\alpha}$ of order $0<\alpha<1$ for an integrable function $f$ is defined by the formula

$$
I^{\alpha}[f](t)=\frac{1}{\Gamma(\alpha)} \int_{c}^{t}(t-s)^{\alpha-1} f(s) d s, t \in[c, d],
$$

where $\Gamma$ is the Euler gamma function. 
The Caputo fractional derivative of order $0<\alpha<1$ of a differentiable function $f$ is defined by the formula

$$
\mathcal{D}_{t}^{\alpha}[f](t)=I^{1-\alpha}\left[f^{\prime}(t)\right]=\frac{1}{\Gamma(1-\alpha)} \int_{c}^{t} \frac{f^{\prime}(s)}{(t-s)^{\alpha}} d s, t \in[c, d] .
$$

Further information on fractional derivatives can be found in [14.

In what follows, we widely use the properties of the Mittag-Leffler type function (see [16]), which is introduced as

$$
E_{\alpha, \beta}(z)=\sum_{m=0}^{\infty} \frac{z^{m}}{\Gamma(\alpha m+\beta)} .
$$

In [25], the following estimate for the Mittag-Leffler function was proved:

$$
\frac{1}{1+\Gamma(1-\alpha) z} \leqslant E_{\alpha, 1}(-z) \leqslant \frac{1}{1+\Gamma(1+\alpha)^{-1} z}, \quad z>0
$$

as $0<\alpha<1$ and this estimate fails for $\alpha \geqslant 1$. Thus, it follows that

$$
0<E_{\alpha, 1}(-z)<1, \quad z>0 \text {. }
$$

Definition 2.2. The space $W^{2,2}[0, \pi]$ is a Hilbert one consisting of all elements of $L^{2}[0, \pi]$ having generalized derivatives up to order 2 in $L^{2}$, i.e.

$$
W^{2,2}[0, \pi]=\left\{f \in L^{2}[0, \pi] \mid f^{\prime}, f^{\prime \prime} \in L^{2}[0, \pi]\right\}
$$

and the norm is defined by

$$
\|f\|_{W^{2,2}[0, \pi]}^{2}=\sum_{i=0}^{2}\left\|\frac{d^{i} f}{d x^{i}}\right\|_{L^{2}[0, \pi]}^{2} .
$$

- $W_{0}^{2,2}[0, \pi]$ is the subspace $W^{2,2}[0, \pi]$ defined as the closure with respect to $\|\cdot\|_{W^{2,2}[0, \pi]}$ of all twice continuously differentiable in $[0, \pi]$ functions vanishing at the points 0 and $\pi$.

- The $L^{2}$-scalar product of two functions $f, g:[0, \pi] \rightarrow \mathbb{R}$ is defined by

$$
(f, g)_{L^{2}}=\int_{0}^{\pi} f(x) \overline{g(x)} d x .
$$

In this section, we study the Cauchy problem for the pseudo-parabolic equation

$$
\begin{aligned}
\mathcal{D}_{t}^{\alpha}[u(t, x) & \left.-u_{x x}(t, x)+\varepsilon u_{x x}(t, \pi-x)\right] \\
& -u_{x x}(t, x)+\varepsilon u_{x x}(t, \pi-x)=f(t, x), \quad(t, x) \in \Omega,
\end{aligned}
$$

with the initial data

$$
u(0, x)=\varphi(x), \quad x \in[0, \pi]
$$

and the homogeneous Dirichlet boundary conditions

$$
u(t, 0)=u(t, \pi)=0,
$$

where $\Omega:=\{0<t<T<\infty, 0<x<\pi\}, f(t, x)$ and $\varphi(x)$ are given functions.

The following statement holds true.

Theorem 2.3. Let $|\varepsilon|<1, f \in C\left([0, T] ; C^{2}[0, \pi]\right)$ and $f(t, 0)=f(t, \pi)=0, \varphi \in C^{4}[0, \pi]$ and $\varphi^{(i)}(0)=\varphi^{(i)}(\pi)=0, i=0,2$. Then there exists a unique regular solution $u \in$ $C^{1}\left([0, T] ; C^{2}[0, \pi]\right)$ of problem (2.2) 2.4 and this solution can be written in the form

$$
u(t, x)=\sum_{k=0}^{\infty} w_{k}(t) \sin (2 k+1) x+\sum_{k=1}^{\infty} v_{k}(t) \sin 2 k x,
$$


where

$$
\begin{aligned}
& w_{k}(t)=\varphi_{1 k} E_{\alpha, 1}\left(-\frac{\lambda_{2 k+1}}{1+\lambda_{2 k+1}} t^{\alpha}\right)-\frac{1}{\lambda_{2 k+1}} \int_{0}^{t} \frac{d}{d s}\left(E_{\alpha, 1}\left(-\frac{\lambda_{2 k+1}}{1+\lambda_{2 k+1}} s^{\alpha}\right)\right) f_{k}^{1}(t-s) d s \\
& v_{k}(t)=\varphi_{2 k} E_{\alpha, 1}\left(-\frac{\lambda_{2 k}}{1+\lambda_{2 k}} t^{\alpha}\right)-\frac{1}{\lambda_{2 k}} \int_{0}^{t} \frac{d}{d s}\left(E_{\alpha, 1}\left(-\frac{\lambda_{2 k}}{1+\lambda_{2 k}} s^{\alpha}\right)\right) f_{k}^{2}(t-s) d s
\end{aligned}
$$

for $t \in[0, T]$ and for all $k \in \mathbb{N}$, where

$$
\begin{array}{ll}
\varphi_{1 k}=\left(\varphi, u_{2 k+1}\right)_{L^{2}}, & \varphi_{2 k}=\left(\varphi, u_{2 k}\right)_{L^{2}}, \\
f_{k}^{1}(t)=\left(f(t, \cdot), u_{2 k+1}\right)_{L^{2}}, & f_{k}^{2}(t)=\left(f(t, \cdot), u_{2 k}\right)_{L^{2}} .
\end{array}
$$

Proof. We begin with proving the existence. Since the system of eigenfunctions (1.4) is an orthonormal basis in $L^{2}(0, \pi)$, we seek the function $u(t, x)$ in the form

$$
u(t, x)=\sum_{k=0}^{\infty} w_{k}(t) \sin (2 k+1) x+\sum_{k=1}^{\infty} v_{k}(t) \sin 2 k x
$$

where $w_{k}(t)$ and $v_{k}(t)$ are unknown functions. Substituting equation (2.5) into equations (2.2), (2.3), we obtain the following equations corresponding to the functions $w_{k}(t)$ and $v_{k}(t)$ :

$$
\begin{aligned}
& \mathcal{D}_{t}^{\alpha} w_{k}(t)+\frac{\lambda_{2 k+1}}{1+\lambda_{2 k+1}} w_{k}(t)=\frac{f_{k}^{1}(t)}{1+\lambda_{2 k+1}}, \quad k \in \mathbb{N}_{0}, \\
& w_{k}(0)=\varphi_{1 k}, \quad k \in \mathbb{N}_{0}
\end{aligned}
$$

and

$$
\begin{aligned}
& \mathcal{D}_{t}^{\alpha} v_{k}(t)+\frac{\lambda_{2 k}}{1+\lambda_{2 k}} v_{k}(t)=\frac{f_{k}^{2}(t)}{1+\lambda_{2 k}}, \quad k \in \mathbb{N}, \\
& v_{k}(0)=\varphi_{2 k}, \quad k \in \mathbb{N} .
\end{aligned}
$$

According to [16], the solutions of equations (2.6) and (2.8) satisfying initial conditions (2.7) and 2.9 can be represented in the form

$$
w_{k}(t)=\varphi_{1 k} E_{\alpha, 1}\left(-\frac{\lambda_{2 k+1}}{1+\lambda_{2 k+1}} t^{\alpha}\right)-\frac{1}{\lambda_{2 k+1}} \int_{0}^{t} \frac{d}{d s}\left(E_{\alpha, 1}\left(-\frac{\lambda_{2 k+1}}{1+\lambda_{2 k+1}} s^{\alpha}\right)\right) f_{k}^{1}(t-s) d s
$$

and

$$
v_{k}(t)=\varphi_{2 k} E_{\alpha, 1}\left(-\frac{\lambda_{2 k}}{1+\lambda_{2 k}} t^{\alpha}\right)-\frac{1}{\lambda_{2 k}} \int_{0}^{t} \frac{d}{d s}\left(E_{\alpha, 1}\left(-\frac{\lambda_{2 k}}{1+\lambda_{2 k}} s^{\alpha}\right)\right) f_{k}^{2}(t-s) d s,
$$

for $t \in[0, T]$ and for all $k \in \mathbb{N}$. Substituting (2.10) and (2.11) into (2.5), we obtain a solution of problem (2.2)-2.4).

Now we are going to prove the convergence of the obtained infinite series corresponding to the functions $u(t, x), \mathcal{D}_{t}^{\alpha} u(t, x), u_{x x}(t, x)$ and $\mathcal{D}_{t}^{\alpha} u_{x x}(t, x)$.

By the assumptions of the theorem we have

$$
\varphi^{(i)}(0)=0, \quad \varphi^{(i)}(\pi)=0, \quad i=0,2, \quad f(t, 0)=f(t, \pi)=0 .
$$


This implies that

$$
\begin{aligned}
\varphi_{1 k} & =\sqrt{\frac{2}{\pi}} \int_{0}^{\pi} \varphi(x) \sin (2 k+1) x d x \\
& =\frac{1}{(2 k+1)^{4}} \sqrt{\frac{2}{\pi}} \int_{0}^{\pi} \varphi^{(4)}(x) \sin (2 k+1) x d x=\frac{1}{(2 k+1)^{4}} \varphi_{1 k}^{(4)}, \\
\varphi_{2 k} & =\sqrt{\frac{2}{\pi}} \int_{0}^{\pi} \varphi(x) \sin 2 k x d x \\
& =\frac{1}{16 k^{4}} \sqrt{\frac{2}{\pi}} \int_{0}^{\pi} \varphi^{(4)}(x) \sin 2 k x d x=\frac{1}{16 k^{4}} \varphi_{2 k}^{(4)}, \\
f_{k}^{1}(t) & =\sqrt{\frac{2}{\pi}} \int_{0}^{\pi} f(t, x) \sin (2 k+1) x d x \\
& =-\frac{1}{(2 k+1)^{2}} \sqrt{\frac{2}{\pi}} \int_{0}^{\pi} f^{\prime \prime}(t, x) \sin (2 k+1) x d x=-\frac{1}{(2 k+1)^{2}} f_{k}^{1(2)}(t), \\
f_{k}^{2}(t) & =\sqrt{\frac{2}{\pi}} \int_{0}^{\pi} f(t, x) \sin 2 k x d x \\
& =-\frac{1}{4 k^{2}} \sqrt{\frac{2}{\pi}} \int_{0}^{\pi} f^{\prime \prime}(t, x) \sin 2 k x d x=-\frac{1}{4 k^{2}} f_{k}^{2(2)}(t) .
\end{aligned}
$$

Here $\varphi_{1 k}^{(4)}, \varphi_{2 k}^{(4)}, f_{k}^{1(2)}(t)$ and $f_{k}^{2(2)}(t)$ are

$$
\begin{aligned}
& \varphi_{1 k}^{(4)}=\sqrt{\frac{2}{\pi}} \int_{0}^{\pi} \varphi^{(4)}(x) \sin (2 k+1) x d x, \\
& \varphi_{2 k}^{(4)}=\sqrt{\frac{2}{\pi}} \int_{0}^{\pi} \varphi^{(4)}(x) \sin 2 k x d x, \\
& f_{k}^{1(2)}(t)=\sqrt{\frac{2}{\pi}} \int_{0}^{\pi} f^{\prime \prime}(t, x) \sin (2 k+1) x d x, \\
& f_{k}^{2(2)}(t)=\sqrt{\frac{2}{\pi}} \int_{0}^{\pi} f^{\prime \prime}(t, x) \sin 2 k x d x,
\end{aligned}
$$

where

$$
f^{\prime \prime}(t, x)=\frac{d^{2} f}{d x^{2}}(t, x) .
$$

Our next step is to calculate $\mathcal{D}_{t}^{\alpha} u(t, x), u_{x x}(t, x)$ and $\mathcal{D}_{t}^{\alpha} u_{x x}(t, x)$. We have:

$$
\mathcal{D}_{t}^{\alpha} u(t, x)=\sum_{k=0}^{\infty} \mathcal{D}_{t}^{\alpha} w_{k}(t) \sin (2 k+1) x+\sum_{k=1}^{\infty} \mathcal{D}_{t}^{\alpha} v_{k}(t) \sin 2 k x .
$$

By (2.6) and (2.8) we find $\mathcal{D}_{t}^{\alpha} w_{k}(t)$ and $\mathcal{D}_{t}^{\alpha} v_{k}(t)$ :

$$
\mathcal{D}_{t}^{\alpha} w_{k}(t)=\frac{f_{k}^{1}(t)}{1+\lambda_{2 k+1}}-\frac{\lambda_{2 k+1}}{1+\lambda_{2 k+1}} w_{k}(t)
$$

and

$$
\mathcal{D}_{t}^{\alpha} v_{k}(t)=\frac{f_{k}^{2}(t)}{1+\lambda_{2 k}}-\frac{\lambda_{2 k}}{1+\lambda_{2 k}} v_{k}(t)
$$


Substituting (2.15), 2.16) into (2.14) and taking into consideration formulae (2.10), (2.11), (2.12), 2.13), we get:

$$
\begin{aligned}
\mathcal{D}_{t}^{\alpha} u(t, x)= & -\sum_{k=0}^{\infty} \frac{f_{k}^{1(2)}(t)}{\left(1+\lambda_{2 k+1}\right)(2 k+1)^{2}} \sin (2 k+1) x-\sum_{k=1}^{\infty} \frac{f_{k}^{2(2)}(t)}{\left(1+\lambda_{2 k}\right) 4 k^{2}} \sin 2 k x \\
& -\sum_{k=0}^{\infty} \frac{1-\varepsilon}{\left(1+\lambda_{2 k+1}\right)(2 k+1)^{2}} \varphi_{1 k}^{(4)} E_{\alpha, 1}\left(-\frac{\lambda_{2 k+1}}{1+\lambda_{2 k+1}} t^{\alpha}\right) \sin (2 k+1) x \\
& -\sum_{k=0}^{\infty} \frac{\sin (2 k+1) x}{\left(1+\lambda_{2 k+1}\right)(2 k+1)^{2}} \int_{0}^{t} \frac{d}{d s}\left(E_{\alpha, 1}\left(-\frac{\lambda_{2 k+1}}{1+\lambda_{2 k+1}} s^{\alpha}\right)\right) f_{k}^{1(2)}(t-s) d s \\
& -\sum_{k=1}^{\infty} \frac{(1+\varepsilon) \sin 2 k x}{\left(1+\lambda_{2 k}\right) 4 k^{2}} \varphi_{2 k}^{(4)} E_{\alpha, 1}\left(-\frac{\lambda_{2 k}}{1+\lambda_{2 k}} t^{\alpha}\right) \\
& -\sum_{k=1}^{\infty} \frac{\sin 2 k x}{\left(1+\lambda_{2 k}\right) 4 k^{2}} \int_{0}^{t} \frac{d}{d s}\left(E_{\alpha, 1}\left(-\frac{\lambda_{2 k}}{1+\lambda_{2 k}} s^{\alpha}\right)\right) f_{k}^{2(2)}(t-s) d s .
\end{aligned}
$$

Applying the operator $\frac{\partial^{2}}{\partial x^{2}}$ to (2.5) and taking into consideration formulae $(2.10),(2.11),(2.12)$, (2.13), we have

$$
\begin{aligned}
u_{x x}(t, x)= & -\sum_{k=0}^{\infty} \frac{\varphi_{1 k}^{(4)}}{(2 k+1)^{2}} E_{\alpha, 1}\left(-\frac{\lambda_{2 k+1}}{1+\lambda_{2 k+1}} t^{\alpha}\right) \sin (2 k+1) x \\
& +\sum_{k=0}^{\infty} \frac{\sin (2 k+1) x}{\lambda_{2 k+1}} \int_{0}^{t} \frac{d}{d s}\left(E_{\alpha, 1}\left(-\frac{\lambda_{2 k+1}}{1+\lambda_{2 k+1}} s^{\alpha}\right)\right) f_{k}^{1(2)}(t-s) d s \\
& -\sum_{k=1}^{\infty} \frac{\varphi_{2 k}^{(4)}}{4 k^{2}} E_{\alpha, 1}\left(-\frac{\lambda_{2 k}}{1+\lambda_{2 k}} t^{\alpha}\right) \sin 2 k x \\
& +\sum_{k=1}^{\infty} \frac{\sin 2 k x}{\lambda_{2 k}} \int_{0}^{t} \frac{d}{d s}\left(E_{\alpha, 1}\left(-\frac{\lambda_{2 k}}{1+\lambda_{2 k}} s^{\alpha}\right)\right) f_{k}^{2(2)}(t-s) d s .
\end{aligned}
$$

Finally, applying the operator $\frac{\partial^{2}}{\partial x^{2}}$ to 2.17), we have

$$
\begin{aligned}
& \mathcal{D}_{t}^{\alpha} u_{x x}(t, x)=\sum_{k=0}^{\infty} \frac{f_{k}^{1(2)}(t)}{1+\lambda_{2 k+1}} \sin (2 k+1) x+\sum_{k=1}^{\infty} \frac{f_{k}^{2(2)}(t)}{1+\lambda_{2 k}} \sin 2 k x \\
& +\sum_{k=0}^{\infty} \frac{1-\varepsilon}{1+\lambda_{2 k+1}} \varphi_{1 k}^{(4)} E_{\alpha, 1}\left(-\frac{\lambda_{2 k+1}}{1+\lambda_{2 k+1}} t^{\alpha}\right) \sin (2 k+1) x \\
& +\sum_{k=0}^{\infty}\left[\frac{1}{1+\lambda_{2 k+1}} \int_{0}^{t} \frac{d}{d s}\left(E_{\alpha, 1}\left(-\frac{\lambda_{2 k+1}}{1+\lambda_{2 k+1}} s^{\alpha}\right)\right) f_{k}^{1(2)}(t-s) d s\right] \sin (2 k+1) x \\
& +\sum_{k=1}^{\infty} \frac{1+\varepsilon}{1+\lambda_{2 k}} \varphi_{2 k}^{(4)} E_{\alpha, 1}\left(-\frac{\lambda_{2 k}}{1+\lambda_{2 k}} t^{\alpha}\right) \sin 2 k x \\
& +\sum_{k=1}^{\infty}\left[\frac{1}{1+\lambda_{2 k}} \int_{0}^{t} \frac{d}{d s}\left(E_{\alpha, 1}\left(-\frac{\lambda_{2 k}}{1+\lambda_{2 k}} s^{\alpha}\right)\right) f_{k}^{2(2)}(t-s) d s\right] \sin 2 k x .
\end{aligned}
$$


Let us to show the convergence of the integral term:

$$
\begin{aligned}
\mid \int_{0}^{t} \frac{d}{d s} & \left(E_{\alpha, 1}\left(-\frac{\lambda_{\xi}}{1+\lambda_{\xi}} s^{\alpha}\right)\right) f_{k}^{i}(t-s) d s \mid \\
& \leqslant \int_{0}^{t}\left|\frac{d}{d s}\left(E_{\alpha, 1}\left(-\frac{\lambda_{\xi}}{1+\lambda_{\xi}} s^{\alpha}\right)\right)\right|\left|f_{k}^{i}(t-s)\right| d s \\
& \leqslant \max _{0 \leqslant s \leqslant t}\left|f_{k}^{i}(t-s)\right| \int_{0}^{t}\left|\frac{d}{d s}\left(E_{\alpha, 1}\left(-\frac{\lambda_{\xi}}{1+\lambda_{\xi}} s^{\alpha}\right)\right)\right| d s \\
& \leqslant \max _{0 \leqslant t \leqslant T}\left|f_{k}^{i}(t)\right| \int_{0}^{t}\left|\frac{d}{d s}\left(E_{\alpha, 1}\left(-\frac{\lambda_{\xi}}{1+\lambda_{\xi}} s^{\alpha}\right)\right)\right| d s .
\end{aligned}
$$

Hereinafter we suppose that $i=1,2$ and if $i=1$, then $\xi=2 k+1, k \in \mathbb{N}_{0}$, while if $i=2$, then $\xi=2 k, k \in \mathbb{N}$. In the above estimates, the integrand is the derivative of the Mittag-Leffler function, which is a non-positive function since

$$
\frac{d}{d s}\left(E_{\alpha, 1}\left(-\frac{\lambda_{\xi}}{1+\lambda_{\xi}} s^{\alpha}\right)\right)=-\frac{\lambda_{\xi}}{1+\lambda_{\xi}} s^{\alpha-1} E_{\alpha, \alpha}\left(-\frac{\lambda_{\xi}}{1+\lambda_{\xi}} s^{\alpha}\right) \leqslant 0 .
$$

It is known that

$$
E_{\alpha, \alpha}(-z)>0, \quad z>0,
$$

see, for instance, [20]. Hence,

$$
\int_{0}^{t}\left|\frac{d}{d s}\left(E_{\alpha, 1}\left(-\frac{\lambda_{\xi}}{1+\lambda_{\xi}} s^{\alpha}\right)\right)\right| d s=\left|\int_{0}^{t} \frac{d}{d s}\left(E_{\alpha, 1}\left(-\frac{\lambda_{\xi}}{1+\lambda_{\xi}} s^{\alpha}\right)\right) d s\right|
$$

Substituting the latter relations into 2.20 and employing (2.1), we obtain:

$$
\begin{aligned}
\left|\int_{0}^{t} \frac{d}{d s}\left(E_{\alpha, 1}\left(-\frac{\lambda_{\xi}}{1+\lambda_{\xi}} s^{\alpha}\right)\right) f_{k}^{i}(t-s) d s\right| & =\max _{0 \leqslant t \leqslant T}\left|f_{k}^{i}(t)\right|\left(1-E_{\alpha, 1}\left(-\frac{\lambda_{\xi}}{1+\lambda_{\xi}} t^{\alpha}\right)\right) \\
& \leqslant C \max _{0 \leqslant t \leqslant T}\left|f_{k}^{i}(t)\right| .
\end{aligned}
$$

Using the estimates (2.1), 2.23) and taking into consideration the formulae (2.12), (2.13), we arrive at the following inequality:

$$
\begin{aligned}
\|u\|_{C_{t, x}(\bar{\Omega})} \leqslant & C \sum_{k=0}^{\infty} \frac{\left|\varphi_{1 k}^{(4)}\right|}{(2 k+1)^{4}}+C \sum_{k=1}^{\infty} \frac{\left|\varphi_{2 k}^{(4)}\right|}{16 k^{4}} \\
& +C \max _{0 \leqslant t \leqslant T} \sum_{k=0}^{\infty} \frac{\left|f_{k}^{1(2)}(t)\right|}{\lambda_{2 k+1}(2 k+1)^{2}}+C \max _{0 \leqslant t \leqslant T} \sum_{k=1}^{\infty} \frac{\left|f_{k}^{2(2)}(t)\right|}{\lambda_{2 k} 4 k^{2}} .
\end{aligned}
$$

By formula (2.17), we have

$$
\begin{aligned}
\left\|\mathcal{D}_{t}^{\alpha} u\right\|_{C_{t, x}(\bar{\Omega})} \leqslant & C \sum_{k=0}^{\infty} \frac{\left|\varphi_{1 k}^{(4)}\right|}{\left(1+\lambda_{2 k+1}\right)(2 k+1)^{2}}+C \sum_{k=1}^{\infty} \frac{\left|\varphi_{2 k}^{(4)}\right|}{\left(1+\lambda_{2 k}\right) 4 k^{2}} \\
& +C \max _{0 \leqslant t \leqslant T} \sum_{k=0}^{\infty} \frac{\left|f_{k}^{1(2)}(t)\right|}{\left(1+\lambda_{2 k+1}\right)(2 k+1)^{2}}+C \max _{0 \leqslant t \leqslant T} \sum_{k=1}^{\infty} \frac{\left|f_{k}^{2(2)}(t)\right|}{\left(1+\lambda_{2 k}\right) 4 k^{2}} .
\end{aligned}
$$


It follows from formula $(2.18)$ that

$$
\begin{aligned}
\left\|u_{x x}\right\|_{C_{t, x}(\bar{\Omega})} \leqslant & C \sum_{k=0}^{\infty} \frac{\left|\varphi_{1 k}^{(4)}\right|}{(2 k+1)^{2}}+C \sum_{k=1}^{\infty} \frac{\left|\varphi_{2 k}^{(4)}\right|}{4 k^{2}} \\
& +C \max _{0 \leqslant t \leqslant T} \sum_{k=0}^{\infty} \frac{\left|f_{k}^{1(2)}(t)\right|}{\lambda_{2 k+1}}+C \max _{0 \leqslant t \leqslant T} \sum_{k=1}^{\infty} \frac{\left|f_{k}^{2(2)}(t)\right|}{\lambda_{2 k}},
\end{aligned}
$$

and by the formula (2.19), we have

$$
\begin{aligned}
\left\|\mathcal{D}_{t}^{\alpha} u_{x x}\right\|_{C_{t, x}(\bar{\Omega})} \leqslant & C \sum_{k=0}^{\infty} \frac{\left|\varphi_{1 k}^{(4)}\right|}{1+\lambda_{2 k+1}}+C \sum_{k=1}^{\infty} \frac{\left|\varphi_{2 k}^{(4)}\right|}{1+\lambda_{2 k}} \\
& +C \max _{0 \leqslant t \leqslant T} \sum_{k=0}^{\infty} \frac{\left|f_{k}^{1(2)}(t)\right|}{1+\lambda_{2 k+1}}+C \max _{0 \leqslant t \leqslant T} \sum_{k=1}^{\infty} \frac{\left|f_{k}^{2(2)}(t)\right|}{1+\lambda_{2 k}} .
\end{aligned}
$$

Hence, series (2.24), 2.25), 2.26) and (2.27) converge absolutely and uniformly in the domain $\bar{\Omega}$.

Now we are in a position to show the uniqueness of the solutions. Let $w(t, x)$ and $v(x, t)$ be solutions of problem $(2.2)-(2.4)$, that is,

$\mathcal{D}_{t}^{\alpha}\left[w(t, x)-w_{x x}(t, x)+\varepsilon w_{x x}(t, \pi-x)\right]-w_{x x}(t, x)+\varepsilon w_{x x}(t, \pi-x)=f(t, x), \quad w(0, x)=\varphi(x)$, $\mathcal{D}_{t}^{\alpha}\left[v(t, x)-v_{x x}(t, x)+\varepsilon v_{x x}(t, \pi-x)\right]-v_{x x}(t, x)+\varepsilon v_{x x}(t, \pi-x)=f(t, x), \quad v(0, x)=\varphi(x)$.

By subtracting these equations one from the other and denoting $u(t, x)=w(t, x)-v(t, x)$, we obtain that

$$
\begin{gathered}
\mathcal{D}_{t}^{\alpha}\left[u(t, x)-u_{x x}(t, x)+\varepsilon u_{x x}(t, \pi-x)\right]-u_{x x}(t, x)+\varepsilon u_{x x}(t, \pi-x)=0, \\
u(0, x)=0 .
\end{gathered}
$$

We also have

$$
\begin{aligned}
w_{k}(t) & =\sqrt{\frac{2}{\pi}} \int_{0}^{\pi} u(t, x) \sin (2 k+1) x d x, \quad k \in \mathbb{N}_{0}, \\
v_{k}(t) & =\sqrt{\frac{2}{\pi}} \int_{0}^{\pi} u(t, x) \sin 2 k x d x, \quad k \in \mathbb{N} .
\end{aligned}
$$

Applying the operator $\mathcal{D}_{t}^{\alpha}$ to 2.30 , we obtain

$$
\begin{array}{lll}
\mathcal{D}_{t}^{\alpha} w_{k}(t)=\sqrt{\frac{2}{\pi}} \int_{0}^{\pi} \mathcal{D}_{t}^{\alpha} u(t, x) \sin (2 k+1) x d x, & & k \in \mathbb{N}_{0}, \\
\mathcal{D}_{t}^{\alpha} v_{k}(t)=\sqrt{\frac{2}{\pi}} \int_{0}^{\pi} \mathcal{D}_{t}^{\alpha} u(t, x) \sin 2 k x d x, & k \in \mathbb{N} .
\end{array}
$$

We multiply both sides of equations $2.28,2.29$ by the functions $\sin (2 k+1) x, \sin 2 k x$ and integrate in the variable $x$ from 0 to $\pi$. Taking into consideration the self-adjointness of the nonlocal operator $\mathcal{L}$, and by $(2.30)-(2.31)$, we have

$$
\begin{gathered}
\mathcal{D}_{t}^{\alpha} w_{k}(t)+\frac{\lambda_{2 k+1}}{1+\lambda_{2 k+1}} w_{k}(t)=0, \quad k \in \mathbb{N}_{0}, \\
w_{k}(0)=0, \quad k \in \mathbb{N}_{0},
\end{gathered}
$$


and

$$
\begin{gathered}
\mathcal{D}_{t}^{\alpha} v_{k}(t)+\frac{\lambda_{2 k}}{1+\lambda_{2 k}} v_{k}(t)=0, \quad k \in \mathbb{N}, \\
v_{k}(0)=0, \quad k \in \mathbb{N} .
\end{gathered}
$$

By formulae (2.10), (2.11) with $\varphi_{1 k}=0, f_{k}^{1}(t)=0, \varphi_{2 k}=0, f_{k}^{2}(t)=0$, the solutions of problem (2.32), 2.33) and (2.34), 2.35) read as $w_{k}(t)=v_{k}(t) \equiv 0$. Hence, by the completeness of the system $u_{2 k}(x), u_{2 k+1}(x)$ in $L^{2}$, we obtain $u(x, t) \equiv 0$.

2.1. Generalized solution. In this section we provide a solution to the direct problem with data from Sobolev spaces.

Theorem 2.4. Let $|\varepsilon|<1, f \in C\left([0, T] ; L^{2}[0, \pi]\right)$ and $\varphi \in W_{0}^{2,2}[0, \pi]$. Then there exists a unique generalized solution $u \in C^{1}\left([0, T] ; W_{0}^{2,2}[0, \pi]\right)$ of problem $(2.2)-(2.4)$ and it can be written as

$$
u(t, x)=\sum_{k=0}^{\infty} w_{k}(t) \sin (2 k+1) x+\sum_{k=1}^{\infty} v_{k}(t) \sin 2 k x
$$

where

$$
w_{k}(t)=\varphi_{1 k} E_{\alpha, 1}\left(-\frac{\lambda_{2 k+1}}{1+\lambda_{2 k+1}} t^{\alpha}\right)+\frac{1}{\lambda_{2 k+1}} \int_{0}^{t} \frac{d}{d s}\left(E_{\alpha, 1}\left(-\frac{\lambda_{2 k+1}}{1+\lambda_{2 k+1}} s^{\alpha}\right)\right) f_{k}^{1}(t-s) d s,
$$

and

$$
v_{k}(t)=\varphi_{2 k} E_{\alpha, 1}\left(-\frac{\lambda_{2 k}}{1+\lambda_{2 k}} t^{\alpha}\right)+\frac{1}{\lambda_{2 k}} \int_{0}^{t} \frac{d}{d s}\left(E_{\alpha, 1}\left(-\frac{\lambda_{2 k}}{1+\lambda_{2 k}} s^{\alpha}\right)\right) f_{k}^{2}(t-s) d s,
$$

for all $t \in[0, T]$ and for each $k \in \mathbb{N}$, where

$$
\begin{array}{ll}
\varphi_{1 k}=\left(\varphi, u_{2 k+1}\right)_{L^{2}}, & \varphi_{2 k}=\left(\varphi, u_{2 k}\right)_{L^{2}}, \\
f_{k}^{1}(t)=\left(f(t, \cdot), u_{2 k+1}\right)_{L^{2}}, & f_{k}^{2}(t)=\left(f(t, \cdot), u_{2 k}\right)_{L^{2}} .
\end{array}
$$

Proof. Using property (2.1), we get the following estimates:

$$
\begin{aligned}
& \|u\|_{C\left([0, T], L^{2}[0, \pi]\right)}^{2} \leqslant C\|\varphi\|_{L^{2}[0, \pi]}^{2}+C \max _{0 \leqslant t \leqslant T} \sum_{k=0}^{\infty} \frac{\left|f_{k}^{1}(t)\right|^{2}}{\lambda_{2 k+1}^{2}}+C \max _{0 \leqslant t \leqslant T} \sum_{k=1}^{\infty} \frac{\left|f_{k}^{2}(t)\right|^{2}}{\lambda_{2 k}^{2}}, \\
& \left\|\mathcal{D}_{t}^{\alpha} u\right\|_{C\left([0, T], L^{2}[0, \pi]\right)}^{2} \leqslant C\|\varphi\|_{L^{2}[0, \pi]}^{2}+C \max _{0 \leqslant t \leqslant T} \sum_{k=0}^{\infty} \frac{\left|f_{k}^{1}(t)\right|^{2}}{\left(1+\lambda_{2 k+1}\right)^{2}}+C \max _{0 \leqslant t \leqslant T} \sum_{k=1}^{\infty} \frac{\left|f_{k}^{2}(t)\right|^{2}}{\left(1+\lambda_{2 k}\right)^{2}}, \\
& \left\|u_{x x}\right\|_{C\left([0, T], L^{2}[0, \pi]\right)}^{2} \leqslant C\left\|\varphi_{x x}\right\|_{L^{2}[0, \pi]}^{2}+\frac{C}{(1-|\varepsilon|)^{2}}\|f\|_{C\left([0, T], L^{2}[0, \pi]\right)}^{2}, \\
& \left\|\mathcal{D}_{t}^{\alpha} u_{x x}\right\|_{C\left([0, T], L^{2}[0, \pi]\right)}^{2} \leqslant C\left\|\varphi_{x x}\right\|_{L^{2}[0, \pi]}^{2}+C\|f\|_{C\left([0, T], L^{2}[0, \pi]\right)}^{2} .
\end{aligned}
$$

Arguing as in the proof of Theorem 2.3, we complete the proof.

\section{INVERSE PROBLEM}

In this Section, we study an inverse problem for the pseudo-parabolic equation (1.1).

Problem 3.1. Find a pair of functions $(u(t, x), f(x))$ satisfying equation (1.1), the homogeneous Dirichlet boundary conditions

$$
u(t, 0)=u(t, \pi)=0,
$$

and an initial condition

$$
u(0, x)=\varphi(x), \quad x \in[0, \pi]
$$


with an additional condition

$$
u(T, x)=\psi(x), \quad x \in[0, \pi],
$$

where $\varphi(x)$ and $\psi(x)$ are sufficiently smooth given functions.

Using the Fourier method, one can check the unique solvability of this problem. A regular solution of problem 3.1 is a pair of functions $(u(t, x), f(x))$, where $u \in C^{1}\left([0, T], C^{2}([0, \pi])\right)$ and $f \in C^{2}([0, \pi])$. A generalized solution of problem 3.1 is a pair of functions $(u(t, x), f(x))$, where $u \in C^{1}\left([0, T], W^{2,2}([0, \pi])\right)$ and $f \in L^{2}([0, \pi])$.

3.1. Main results. For equation (1.1) with conditions (3.1)-(3.3) the following theorem holds true.

Theorem 3.2. Let $T>0,|\varepsilon|<1, \varphi, \psi \in C^{4}[0, \pi]$ and

$$
\varphi^{(i)}(0)=\varphi^{(i)}(\pi)=\psi^{(i)}(0)=\psi^{(i)}(\pi)=0, \quad i=0,2 .
$$

Then there exists a unique regular solution $u \in C^{1}\left([0, T], C^{2}[0, \pi]\right), f \in C^{2}[0, \pi]$ of problem 3.1 and it can be written as

$$
\begin{aligned}
u(t, x)= & \varphi(x)+\sum_{k=0}^{\infty} \frac{\left(1-E_{\alpha, 1}\left(-\frac{\lambda_{2 k+1}}{1+\lambda_{2 k+1}} t^{\alpha}\right)\right) \sin (2 k+1) x}{\left(1-E_{\alpha, 1}\left(-\frac{\lambda_{2 k+1}}{1+\lambda_{2 k+1}} T^{\alpha}\right)\right)(2 k+1)^{2}}\left(\varphi_{1 k}^{(2)}-\psi_{1 k}^{(2)}\right) \\
& +\sum_{k=1}^{\infty} \frac{\left(1-E_{\alpha, 1}\left(-\frac{\lambda_{2 k}}{1+\lambda_{2 k}} t^{\alpha}\right)\right) \sin 2 k x}{\left(1-E_{\alpha, 1}\left(-\frac{\lambda_{2 k}}{1+\lambda_{2 k}} T^{\alpha}\right)\right) 4 k^{2}}\left(\varphi_{2 k}^{(2)}-\psi_{2 k}^{(2)}\right), \\
f(x)=- & \varphi_{x x}(x)+\varepsilon \varphi_{x x}(\pi-x)+\sum_{k=0}^{\infty} \frac{(1-\varepsilon)\left(\varphi_{1 k}^{(2)}-\psi_{1 k}^{(2)}\right)}{1-E_{\alpha, 1}\left(-\frac{\lambda_{2 k+1}}{1+\lambda_{2 k+1}} T^{\alpha}\right)} \sin (2 k+1) x \\
& +\sum_{k=1}^{\infty} \frac{(1+\varepsilon)\left(\varphi_{2 k}^{(2)}-\psi_{2 k}^{(2)}\right)}{1-E_{\alpha, 1}\left(-\frac{\lambda_{2 k}}{1+\lambda_{2 k}} T^{\alpha}\right)} \sin 2 k x,
\end{aligned}
$$

for $(t, x) \in \bar{\Omega}$, where

$$
\varphi_{1 k}^{(2)}=\left(\varphi_{x x}, u_{2 k+1}\right)_{L^{2}}, \quad \varphi_{2 k}^{(2)}=\left(\varphi_{x x}, u_{2 k}\right)_{L^{2}}, \quad \psi_{1 k}^{(2)}=\left(\psi_{x x}, u_{2 k+1}\right)_{L^{2}}, \quad \psi_{2 k}^{(2)}=\left(\psi_{x x}, u_{2 k}\right)_{L^{2}} .
$$

Proof. We begin with proving the existence. Since the eigenfunctions (1.4) form an orthonormal basis in $L^{2}[0, \pi]$, we seek functions $u(t, x)$ and $f(x)$ in the form

$$
u(t, x)=\sum_{k=0}^{\infty} w_{k}(t) \sin (2 k+1) x+\sum_{k=1}^{\infty} v_{k}(t) \sin 2 k x,
$$

and

$$
f(x)=\sum_{k=0}^{\infty} f_{k}^{1} \sin (2 k+1) x+\sum_{k=1}^{\infty} f_{k}^{2} \sin 2 k x,
$$

where $w_{k}(t), v_{k}(t), f_{k}^{1}$ and $f_{k}^{2}$ are unknown. Substituting equations (3.4) and (3.5) into equation (1.1), we obtain the following equations for the functions $w_{k}(t), v_{k}(t)$ and the constants $f_{k}^{1}, f_{k}^{2}$ :

$$
\mathcal{D}_{t}^{\alpha} w_{k}(t)+\frac{\lambda_{2 k+1}}{1+\lambda_{2 k+1}} w_{k}(t)=\frac{f_{k}^{1}}{1+\lambda_{2 k+1}}, \quad \mathcal{D}_{t}^{\alpha} v_{k}(t)+\frac{\lambda_{2 k}}{1+\lambda_{2 k}} v_{k}(t)=\frac{f_{k}^{2}}{1+\lambda_{2 k}} .
$$


Solving these equations, we obtain

$$
w_{k}(t)=\frac{f_{k}^{1}}{\lambda_{2 k+1}}+C_{1 k} E_{\alpha, 1}\left(-\frac{\lambda_{2 k+1}}{1+\lambda_{2 k+1}} t^{\alpha}\right), \quad v_{k}(t)=\frac{f_{k}^{2}}{\lambda_{2 k}}+C_{2 k} E_{\alpha, 1}\left(-\frac{\lambda_{2 k}}{1+\lambda_{2 k}} t^{\alpha}\right),
$$

where the constants $f_{k}^{1}, f_{k}^{2}, C_{1 k}$ and $C_{2 k}$ are unknown. To find these constants, we use conditions (3.2) and (3.3). Let

$$
\varphi_{1 k}=\left(\varphi, u_{2 k+1}\right)_{L^{2}}, \quad \varphi_{2 k}=\left(\varphi, u_{2 k}\right)_{L^{2}}, \quad \psi_{1 k}=\left(\psi, u_{2 k+1}\right)_{L^{2}}, \quad \psi_{2 k}=\left(\psi, u_{2 k}\right)_{L^{2}} .
$$

We first find $C_{1 k}$ :

$$
\begin{aligned}
& w_{k}(0)=\frac{f_{k}^{1}}{\lambda_{2 k+1}}+C_{1 k}=\varphi_{1 k} \\
& w_{k}(T)=\frac{f_{k}^{1}}{\lambda_{2 k+1}}+C_{1 k} E_{\alpha, 1}\left(-\frac{\lambda_{2 k+1}}{1+\lambda_{2 k+1}} T^{\alpha}\right)=\psi_{1 k} .
\end{aligned}
$$

Then we have

$$
C_{1 k}=\frac{\varphi_{1 k}-\psi_{1 k}}{1-E_{\alpha, 1}\left(-\frac{\lambda_{2 k+1}}{1+\lambda_{2 k+1}} T^{\alpha}\right)} .
$$

The constant $f_{k}^{1}$ is represented as

$$
f_{k}^{1}=\varphi_{1 k} \lambda_{2 k+1}-C_{1 k} \lambda_{2 k+1}
$$

Now we find $C_{2 k}$ :

$$
\begin{aligned}
& v_{k}(0)=\frac{f_{k}^{2}}{\lambda_{2 k}}+C_{2 k}=\varphi_{2 k}, \\
& v_{k}(T)=\frac{f_{k}^{2}}{\lambda_{2 k}}+C_{2 k} E_{\alpha, 1}\left(-\frac{\lambda_{2 k}}{1+\lambda_{2 k}} T^{\alpha}\right)=\psi_{2 k}, \\
& \varphi_{2 k}-C_{2 k}+C_{2 k} E_{\alpha, 1}\left(-\frac{\lambda_{2 k}}{1+\lambda_{2 k}} T^{\alpha}\right)=\psi_{2 k} .
\end{aligned}
$$

Then we obtain

For the constant $f_{k}^{2}$ we find

$$
C_{2 k}=\frac{\varphi_{2 k}-\psi_{2 k}}{1-E_{\alpha, 1}\left(-\frac{\lambda_{2 k}}{1+\lambda_{2 k}} T^{\alpha}\right)}
$$

$$
f_{k}^{2}=\varphi_{2 k} \lambda_{2 k}-C_{2 k} \lambda_{2 k}
$$

Substituting $w_{k}(t), v_{k}(t), f_{k}^{1}$ and $f_{k}^{2}$ into equations 3.4 and 3.5 , we find

$$
\begin{aligned}
u(t, x)= & \varphi(x)+\sum_{k=0}^{\infty} C_{1 k}\left(E_{\alpha, 1}\left(-\frac{\lambda_{2 k+1}}{1+\lambda_{2 k+1}} t^{\alpha}\right)-1\right) \sin (2 k+1) x \\
& +\sum_{k=1}^{\infty} C_{2 k}\left(E_{\alpha, 1}\left(-\frac{\lambda_{2 k}}{1+\lambda_{2 k}} t^{\alpha}\right)-1\right) \sin 2 k x .
\end{aligned}
$$

By the assumption of the theorem we have

$$
\varphi(0)=\varphi(\pi)=\psi(0)=\psi(\pi)=0 .
$$

This implies

$$
C_{1 k}=\frac{\varphi_{1 k}-\psi_{1 k}}{1-E_{\alpha, 1}\left(-\frac{\lambda_{2 k+1}}{1+\lambda_{2 k+1}} T^{\alpha}\right)}=-\frac{\varphi_{1 k}^{(2)}-\psi_{1 k}^{(2)}}{\left(1-E_{\alpha, 1}\left(-\frac{\lambda_{2 k+1}}{1+\lambda_{2 k+1}} T^{\alpha}\right)\right)(2 k+1)^{2}} .
$$


In the same way we obtain:

$$
C_{2 k}=-\frac{\varphi_{2 k}^{(2)}-\psi_{2 k}^{(2)}}{\left(1-E_{\alpha, 1}\left(-\frac{\lambda_{2 k}}{1+\lambda_{2 k}} T^{\alpha}\right)\right) 4 k^{2}} .
$$

Then we get

$$
\begin{aligned}
u(t, x)=\varphi(x) & +\sum_{k=0}^{\infty} \frac{\left(1-E_{\alpha, 1}\left(-\frac{\lambda_{2 k+1}}{1+\lambda_{2 k+1}} t^{\alpha}\right)\right) \sin (2 k+1) x}{\left(1-E_{\alpha, 1}\left(-\frac{\lambda_{2 k+1}}{1+\lambda_{2 k+1}} T^{\alpha}\right)\right)(2 k+1)^{2}}\left(\varphi_{1 k}^{(2)}-\psi_{1 k}^{(2)}\right) \\
& +\sum_{k=1}^{\infty} \frac{\left(1-E_{\alpha, 1}\left(-\frac{\lambda_{2 k}}{1+\lambda_{2 k}} t^{\alpha}\right)\right) \sin 2 k x}{\left(1-E_{\alpha, 1}\left(-\frac{\lambda_{2 k}}{1+\lambda_{2 k}} T^{\alpha}\right)\right) 4 k^{2}}\left(\varphi_{2 k}^{(2)}-\psi_{2 k}^{(2)}\right) .
\end{aligned}
$$

As above, we also find that

$$
\begin{aligned}
f(x)= & -\varphi_{x x}(x)+\varepsilon \varphi_{x x}(\pi-x)+\sum_{k=0}^{\infty} \frac{(1-\varepsilon)\left(\varphi_{1 k}^{(2)}-\psi_{1 k}^{(2)}\right)}{1-E_{\alpha, 1}\left(-\frac{\lambda_{2 k+1}}{1+\lambda_{2 k+1}} T^{\alpha}\right)} \sin (2 k+1) x \\
& +\sum_{k=1}^{\infty} \frac{(1+\varepsilon)\left(\varphi_{2 k}^{(2)}-\psi_{2 k}^{(2)}\right)}{1-E_{\alpha, 1}\left(-\frac{\lambda_{2 k}}{1+\lambda_{2 k}} T^{\alpha}\right)} \sin 2 k x .
\end{aligned}
$$

The assumption $T>0$ and the estimate (2.1) guarantee that the denominators in formulae (3.6) and (3.7) do not vanish. Moreover, there exists a constant $C>0$ such that

$$
1-E_{\alpha, 1}\left(-\frac{\lambda_{\xi}}{1+\lambda_{\xi}} T^{\alpha}\right)>C>0
$$

for all $\xi \in \mathbb{Z}_{+}$.

Let us calculate $\mathcal{D}_{t}^{\alpha} u(t, x), u_{x x}(t, x)$ and $\mathcal{D}_{t}^{\alpha} u_{x x}(t, x)$. According to [16], we have

$$
\mathcal{D}_{t}^{\alpha}\left(E_{\alpha, 1}\left(-\lambda t^{\alpha}\right)\right)=-\lambda E_{\alpha, 1}\left(-\lambda t^{\alpha}\right) .
$$


Applying the operator $\mathcal{D}_{t}^{\alpha}$ to $(3.6)$ and taking into consideration $(3.9)$, we have

$$
\begin{aligned}
\mathcal{D}_{t}^{\alpha} u(t, x)= & \sum_{k=0}^{\infty} \frac{(1-\varepsilon) E_{\alpha, 1}\left(-\frac{\lambda_{2 k+1}}{1+\lambda_{2 k+1}} t^{\alpha}\right) \sin (2 k+1) x}{\left(1-E_{\alpha, 1}\left(-\frac{\lambda_{2 k+1}}{1+\lambda_{2 k+1}} T^{\alpha}\right)\right)\left(1+\lambda_{2 k+1}\right)}\left(\varphi_{1 k}^{(2)}-\psi_{1 k}^{(2)}\right) \\
& +\sum_{k=1}^{\infty} \frac{(1+\varepsilon) E_{\alpha, 1}\left(-\frac{\lambda_{2 k}}{1+\lambda_{2 k}} t^{\alpha}\right) \sin 2 k x}{\left(1-E_{\alpha, 1}\left(-\frac{\lambda_{2 k}}{1+\lambda_{2 k}} T^{\alpha}\right)\right)\left(1+\lambda_{2 k}\right)}\left(\varphi_{2 k}^{(2)}-\psi_{2 k}^{(2)}\right), \\
u_{x x}(t, x)= & \varphi_{x x}(x)-\sum_{k=0}^{\infty} \frac{\left(1-E_{\alpha, 1}\left(-\frac{\lambda_{2 k+1}}{1+\lambda_{2 k+1}} t^{\alpha}\right)\right) \sin (2 k+1) x}{1-E_{\alpha, 1}\left(-\frac{\lambda_{2 k+1}}{1+\lambda_{2 k+1}} T^{\alpha}\right)}\left(\varphi_{1 k}^{(2)}-\psi_{1 k}^{(2)}\right) \\
& -\sum_{k=1}^{\infty} \frac{\left(1-E_{\alpha, 1}\left(-\frac{\lambda_{2 k}}{1+\lambda_{2 k}} t^{\alpha}\right)\right) \sin 2 k x}{1-E_{\alpha, 1}\left(-\frac{\lambda_{2 k}}{1+\lambda_{2 k}} T^{\alpha}\right)}\left(\varphi_{2 k}^{(2)}-\psi_{2 k}^{(2)}\right),
\end{aligned}
$$

and we also have

$$
\begin{aligned}
\mathcal{D}_{t}^{\alpha} u_{x x}(t, x)= & -\sum_{k=0}^{\infty} \frac{\lambda_{2 k+1} E_{\alpha, 1}\left(-\frac{\lambda_{2 k+1}}{1+\lambda_{2 k+1}} t^{\alpha}\right) \sin (2 k+1) x}{\left(1-E_{\alpha, 1}\left(-\frac{\lambda_{2 k+1}}{1+\lambda_{2 k+1}} T^{\alpha}\right)\right)\left(1+\lambda_{2 k+1}\right)}\left(\varphi_{1 k}^{(2)}-\psi_{1 k}^{(2)}\right) \\
& -\sum_{k=1}^{\infty} \frac{\lambda_{2 k} E_{\alpha, 1}\left(-\frac{\lambda_{2 k}}{1+\lambda_{2 k}} t^{\alpha}\right) \sin 2 k x}{\left(1-E_{\alpha, 1}\left(-\frac{\lambda_{2 k}}{1+\lambda_{2 k}} T^{\alpha}\right)\right)\left(1+\lambda_{2 k}\right)}\left(\varphi_{2 k}^{(2)}-\psi_{2 k}^{(2)}\right)
\end{aligned}
$$

In view of estimate (2.1), in order to ensure the convergence of series $(3.6),(3.10),(3.11)$, (3.12) and (3.7), we have the following estimate

$$
\|u\|_{C_{t, x}(\bar{\Omega})} \leqslant\|\varphi\|_{C([0, \pi])}+C \sum_{k=0}^{\infty} \frac{\left|\varphi_{1 k}^{(2)}\right|+\left|\psi_{1 k}^{(2)}\right|}{(2 k+1)^{2}}+C \sum_{k=1}^{\infty} \frac{\left|\varphi_{2 k}^{(2)}\right|+\left|\psi_{2 k}^{(2)}\right|}{4 k^{2}} .
$$

By the assumptions of the theorem we have

$$
\begin{array}{lll}
\varphi^{(i)}(0)=0, & \varphi^{(i)}(\pi)=0, & i=0,2, \\
\psi^{(i)}(0)=0, & \psi^{(i)}(\pi)=0, & i=0,2 .
\end{array}
$$

This implies that the coefficients of the function $\psi(x)$ satisfy identities $(2.12)$. Using this fact for $f(x)$, we arrive at the estimate

$$
\|f\|_{C([0, \pi])} \leqslant C\left\|\varphi_{x x}\right\|_{C([0, \pi])}+C \sum_{k=0}^{\infty} \frac{\left|\varphi_{1 k}^{(4)}\right|+\left|\psi_{1 k}^{(4)}\right|}{(2 k+1)^{2}}+C \sum_{k=1}^{\infty} \frac{\left|\varphi_{2 k}^{(4)}\right|+\left|\psi_{2 k}^{(4)}\right|}{4 k^{2}} .
$$


We also have:

$$
\begin{aligned}
& \left\|\mathcal{D}_{t}^{\alpha} u\right\|_{C_{t, x}(\bar{\Omega})} \leqslant C \sum_{k=0}^{\infty} \frac{\left|\varphi_{1 k}^{(2)}\right|+\left|\psi_{1 k}^{(2)}\right|}{1+\lambda_{2 k+1}}+C \sum_{k=1}^{\infty} \frac{\left|\varphi_{2 k}^{(2)}\right|+\left|\psi_{2 k}^{(2)}\right|}{1+\lambda_{2 k}} \\
& \left\|u_{x x}\right\|_{C_{t, x}(\bar{\Omega})} \leqslant C \sum_{k=0}^{\infty} \frac{\left|\varphi_{1 k}^{(4)}\right|+\left|\psi_{1 k}^{(4)}\right|}{(2 k+1)^{2}}+C \sum_{k=1}^{\infty} \frac{\left|\varphi_{2 k}^{(4)}\right|+\left|\psi_{2 k}^{(4)}\right|}{4 k^{2}} \\
& \left\|\mathcal{D}_{t}^{\alpha} u_{x x}\right\|_{C_{t, x}(\bar{\Omega})} \leqslant C \sum_{k=0}^{\infty} \frac{\left|\varphi_{1 k}^{(4)}\right|+\left|\psi_{1 k}^{(4)}\right|}{(2 k+1)^{2}}+C \sum_{k=1}^{\infty} \frac{\left|\varphi_{2 k}^{(4)}\right|+\left|\psi_{2 k}^{(4)}\right|}{4 k^{2}}
\end{aligned}
$$

This completes the proof of the existence of the solution.

We proceed to proving the uniqueness. We suppose that $\left\{u_{1}(t, x), f_{1}(x)\right\}$ and $\left\{u_{2}(t, x), f_{2}(x)\right\}$ are solutions of Problem 3.1. Then $u(t, x)=u_{1}(t, x)-u_{2}(t, x)$ and $f(x)=f_{1}(x)-f_{2}(x)$ satisfy the following problem:

$$
\begin{gathered}
\mathcal{D}_{t}^{\alpha}\left[u(t, x)-u_{x x}(t, x)+\varepsilon u_{x x}(t, \pi-x)\right]-u_{x x}(t, x)+\varepsilon u_{x x}(t, \pi-x)=f(x), \\
u(0, x)=0, \\
u(T, x)=0 .
\end{gathered}
$$

We also have

$$
\begin{aligned}
w_{k}(t) & =\sqrt{\frac{2}{\pi}} \int_{0}^{\pi} u(t, x) \sin (2 k+1) x d x, \quad k \in \mathbb{N}_{0}, \\
v_{k}(t) & =\sqrt{\frac{2}{\pi}} \int_{0}^{\pi} u(t, x) \sin 2 k x d x, \quad k \in \mathbb{N},
\end{aligned}
$$

and

$$
\begin{aligned}
& f_{k}^{1}=\sqrt{\frac{2}{\pi}} \int_{0}^{\pi} f(x) \sin (2 k+1) x d x, \quad k \in \mathbb{N}_{0}, \\
& f_{k}^{2}=\sqrt{\frac{2}{\pi}} \int_{0}^{\pi} f(x) \sin 2 k x d x, \quad k \in \mathbb{N}_{0} .
\end{aligned}
$$

Applying the operator $\mathcal{D}_{t}^{\alpha}$ to 3.18 , we get

$$
\begin{aligned}
& \mathcal{D}_{t}^{\alpha} w_{k}(t)=\sqrt{\frac{2}{\pi}} \int_{0}^{\pi} \mathcal{D}_{t}^{\alpha} u(t, x) \sin (2 k+1) x d x, \quad k \in \mathbb{N}_{0}, \\
& \mathcal{D}_{t}^{\alpha} v_{k}(t)=\sqrt{\frac{2}{\pi}} \int_{0}^{\pi} \mathcal{D}_{t}^{\alpha} u(t, x) \sin 2 k x d x, \quad k \in \mathbb{N} .
\end{aligned}
$$

We multiply both sides of equations (3.15)-(3.17) by the functions $\sin (2 k+1) x$, sin $2 k x$ and integrate in the variable $x$ from 0 to $\pi$. Taking into consideration the self-adjointness of the nonlocal operator $\mathcal{L}$, and by $(3.18)-(3.20)$, we have

$$
\begin{gathered}
\mathcal{D}_{t}^{\alpha} w_{k}(t)+\frac{\lambda_{2 k+1}}{1+\lambda_{2 k+1}} w_{k}(t)=\frac{f_{k}^{1}}{1+\lambda_{2 k+1}}, \quad k \in \mathbb{N}_{0} \\
w_{k}(0)=0, \quad k \in \mathbb{N}_{0}, \\
w_{k}(T)=0, \quad k \in \mathbb{N}_{0},
\end{gathered}
$$


and

$$
\begin{gathered}
\mathcal{D}_{t}^{\alpha} v_{k}(t)+\frac{\lambda_{2 k}}{1+\lambda_{2 k}} v_{k}(t)=\frac{f_{k}^{2}}{1+\lambda_{2 k}}, \quad k \in \mathbb{N}, \\
v_{k}(0)=0, \quad k \in \mathbb{N}, \\
v_{k}(T)=0, \quad k \in \mathbb{N} .
\end{gathered}
$$

Arguing as in the proof of the existence result, we confirm easily that the solutions of equations (3.21) - 3.23) and (3.24)-(3.26) are $w_{k}(t)=v_{k}(t)=f_{k}^{1}=f_{k}^{2}=0$. Then

$$
\begin{aligned}
0 & =\sqrt{\frac{2}{\pi}} \int_{0}^{\pi} u(t, x) \sin (2 k+1) x d x, & & k \in \mathbb{N}_{0}, \\
0 & =\sqrt{\frac{2}{\pi}} \int_{0}^{\pi} u(t, x) \sin 2 k x d x, & & k \in \mathbb{N},
\end{aligned}
$$

and

$$
\begin{aligned}
& 0=\sqrt{\frac{2}{\pi}} \int_{0}^{\pi} f(x) \sin (2 k+1) x d x, \quad k \in \mathbb{N}_{0}, \\
& 0=\sqrt{\frac{2}{\pi}} \int_{0}^{\pi} f(x) \sin 2 k x d x, \quad k \in \mathbb{N}_{0} .
\end{aligned}
$$

Therefore, owing to the completeness of the system $u_{2 k}(x), u_{2 k+1}(x)$ in $L^{2}$, we obtain $u(x, t) \equiv$ 0 and $f(x) \equiv 0$. This prove the uniqueness of the solution of problem 3.1. The proof is complete.

3.2. Generalized solutions. Once we deal with a generalized solution instead of the regular one of problem 3.1. Theorem 3.2 can be modified as follows.

Theorem 3.3. Let $T>0,|\varepsilon|<1$ and $\varphi, \psi \in W_{0}^{2,2}[0, \pi]$. Then there exists a unique generalized solution $u \in C^{1}\left([0, T], W_{0}^{2,2}[0, \pi]\right), f \in L^{2}[0, \pi]$ of problem 3.1 and it can be written as

$$
\begin{aligned}
u(t, x)= & \varphi(x)+\sum_{k=0}^{\infty} \frac{\left(1-E_{\alpha, 1}\left(-\frac{\lambda_{2 k+1}}{1+\lambda_{2 k+1}} t^{\alpha}\right)\right) \sin (2 k+1) x}{\left(1-E_{\alpha, 1}\left(-\frac{\lambda_{2 k+1}}{1+\lambda_{2 k+1}} T^{\alpha}\right)\right)(2 k+1)^{2}}\left(\varphi_{1 k}^{(2)}-\psi_{1 k}^{(2)}\right) \\
& +\sum_{k=1}^{\infty} \frac{\left(1-E_{\alpha, 1}\left(-\frac{\lambda_{2 k}}{1+\lambda_{2 k}} t^{\alpha}\right)\right) \sin 2 k x}{\left(1-E_{\alpha, 1}\left(-\frac{\lambda_{2 k}}{1+\lambda_{2 k}} T^{\alpha}\right)\right) 4 k^{2}}\left(\varphi_{2 k}^{(2)}-\psi_{2 k}^{(2)}\right), \\
f(x)= & -\varphi_{x x}(x)+\varepsilon \varphi_{x x}(\pi-x)+\sum_{k=0}^{\infty} \frac{(1-\varepsilon)\left(\varphi_{1 k}^{(2)}-\psi_{1 k}^{(2)}\right)}{1-E_{\alpha, 1}\left(-\frac{\lambda_{2 k+1}}{1+\lambda_{2 k+1}} T^{\alpha}\right)} \sin (2 k+1) x \\
& +\sum_{k=1}^{\infty} \frac{(1+\varepsilon)\left(\varphi_{2 k}^{(2)}-\psi_{2 k}^{(2)}\right)}{1-E_{\alpha, 1}\left(-\frac{\lambda_{2 k}}{1+\lambda_{2 k}} T^{\alpha}\right)} \sin 2 k x
\end{aligned}
$$


for $(t, x) \in \bar{\Omega}$, where

$$
\begin{array}{ll}
\varphi_{1 k}^{(2)}=\left(\varphi_{x x}, u_{2 k+1}\right)_{L^{2}}, & \varphi_{2 k}^{(2)}=\left(\varphi_{x x}, u_{2 k}\right)_{L^{2}}, \\
\psi_{1 k}^{(2)}=\left(\psi_{x x}, u_{2 k+1}\right)_{L^{2}}, & \psi_{2 k}^{(2)}=\left(\psi_{x x}, u_{2 k}\right)_{L^{2}} .
\end{array}
$$

Proof. Reproducing the proof of Theorem 3.2, we can prove the unique solvability in the considered case, too. We just need to clarify the functional classes. By using Plancherel identity and orthogonality of $u_{2 k}, u_{2 k+1}$, we get:

$$
\begin{aligned}
& \|u\|_{C\left([0, T], L^{2}[0, \pi]\right)}^{2} \leqslant C\|\varphi\|_{L^{2}[0, \pi]}^{2}+C \sum_{k=0}^{\infty} \frac{\left|\varphi_{1 k}^{(2)}\right|^{2}+\left|\psi_{1 k}^{(2)}\right|^{2}}{(2 k+1)^{4}}+C \sum_{k=1}^{\infty} \frac{\left|\varphi_{2 k}^{(2)}\right|^{2}+\left|\psi_{2 k}^{(2)}\right|^{2}}{(2 k)^{4}} \\
& \|f\|_{L^{2}[0, \pi]}^{2} \leqslant C\left\|\varphi_{x x}\right\|_{L^{2}[0, \pi]}^{2}+C\left\|\psi_{x x}\right\|_{L^{2}[0, \pi]}^{2} .
\end{aligned}
$$

Moreover, we find:

$$
\begin{aligned}
& \left\|\mathcal{D}_{t}^{\alpha} u\right\|_{C\left([0, T], L^{2}[0, \pi]\right)}^{2} \leqslant C \sum_{k=0}^{\infty} \frac{\left|\varphi_{1 k}^{(2)}\right|^{2}+\left|\psi_{1 k}^{(2)}\right|^{2}}{\left(1+\lambda_{2 k+1}\right)^{2}}+C \sum_{k=1}^{\infty} \frac{\left|\varphi_{2 k}^{(2)}\right|^{2}+\left|\psi_{2 k}^{(2)}\right|^{2}}{\left(1+\lambda_{2 k}\right)^{2}} \\
& \left\|u_{x x}\right\|_{C\left([0, T], L^{2}[0, \pi]\right)}^{2} \leqslant C\left\|\varphi_{x x}\right\|_{L^{2}[0, \pi]}^{2}+C\left\|\psi_{x x}\right\|_{L^{2}[0, \pi]}^{2}, \\
& \left\|\mathcal{D}_{t}^{\alpha} u_{x x}\right\|_{C\left([0, T], L^{2}[0, \pi]\right)}^{2} \leqslant C\left\|\varphi_{x x}\right\|_{L^{2}[0, \pi]}^{2}+C\left\|\psi_{x x}\right\|_{L^{2}[0, \pi]}^{2} .
\end{aligned}
$$

These estimates allow us to reproduce the arguing from the proof of Theorem 3.2 . The proof is complete.

\section{BIBLIOGRAPHY}

1. B. Ahmad, A. Alsaedi, M. Kirane, R. Tapdigoglu. An inverse problem for space and time fractional evolution equations with an involution perturbation // Quaest. Math. 40:2, 151-160 (2017).

2. N. Al-Salti, M. Kirane, B.T. Torebek. On a class of inverse problems for a heat equation with involution perturbation // Hacet. J. Math. Stat. 48:3, 669-681 (2019).

3. T.B. Benjamin, J.L. Bona, J.J. Mahony. Model equations for long waves in nonlinear dispersive systems // Philosoph. Trans. Royal Soc. A: Mathem. Phys. Engineer. Sci. 272:1220, 47-78 (1972).

4. G.I. Barenblatt, J. Garcia-Azorero, A. De Pablo, J.L. Vazquez. Mathematical model of the nonequilibrium water-oil displacement in porous strata // Appl. Anal. 65:1-2, 19-45 (1997).

5. P.J. Chen, M.E. Gurtin. On a theory of heat conduction involving two temperatures // Z. Angew. Math. Phys. 19:4, 614-627 (1968).

6. G.V. Demidenko, S.V. Uspenskii. Partial differential equations and systems not solvable with respect to the highest-order derivative. Nauchnaya Kniga, Novosibirsk (1998). [Marcel Dekker, New York (2003).]

7. C. Fetecau, C-a. Fetecau, M. Kamran, D. Vieru. Exact solutions for the flow of a generalized Oldroyd-B fluid induced by aconstantly accelerating plate between two side walls perpendicular to the plate // J. Non-Newtonian Fluid Mech. 156:3, 189-201 (2009).

8. V.E. Fedorov, R.R. Nazhimov. Inverse problems for a class of degenerate evolution equations with Riemann-Liouville derivative // Fract. Calc. Appl. Anal. 22:2, 271-286 (2019).

9. A. Hazanee, D. Lesnic, M.I. Ismailov, N.B. Kerimov. Inverse time-dependent source problems for the heat equation with nonlocal boundary conditions // Appl. Math. Comput. 346, 800-815 (2019).

10. M. Kirane, N. Al-Salti. Inverse problems for a nonlocal wave equation with an involution perturbation // J. Nonl. Sci. Appl. 9:3, 1243-1251 (2016).

11. N. Kinash, J. Janno. Inverse problems for a perturbed time fractional diffusion equation with final overdetermination // Math. Meth. Appl. Sci. 41:5, 1925-1943 (2018).

12. I.A. Kaliev, M.M. Sabitova. Problems of determining the temperature and density of heat sources from the initial and final temperatures // J. Appl. Ind. Math. 4:3, 332-339 (2010). 
13. M. Kirane, B. Samet, B.T. Torebek. Determination of an unknown source term temperature distribution for the sub-diffusion equation at the initial and final data // Electron. J. Diff. Equat. 2017:257, 1-13 (2017).

14. A.A. Kilbas, H.M. Srivastava, J.J. Trujillo. Theory and applications of fractional differential equations. North-Holland: Elsevier (2006).

15. Y. Kian, M. Yamamoto. Reconstruction and stable recovery of source terms and coefficients appearing in diffusion equations // Inverse Probl. 35:11, 115006 (2019).

16. Y. Luchko, R. Gorenflo. An operational method for solving fractional differential equations with the Caputo derivatives // Acta Math. Vietnam. 24:2, 207-233 (1999).

17. A.S. Lyubanova, A. Tani. An inverse problem for pseudo-parabolic equation of filtration. The existence, uniqueness and regularity // Appl. Anal. 90:10, 1557-1571 (2011).

18. A.S. Lyubanova, A. Tani. On inverse problems for pseudoparabolic and parabolic equations of filtration // Inverse Probl. Sci. Eng. 19:7, 1023-1042 (2011).

19. A.S. Lyubanova, A.V. Velisevich. Inverse problems for the stationary and pseudoparabolic equations of diffusion // Appl. Anal. 98:11, 1997-2010 (2019).

20. J.J. Nieto. Maximum principles for fractional differential equations derived from Mittag-Leffler functions // Appl. Math. Lett. 23:10, 1248-1251 (2010).

21. I. Orazov, M.A. Sadybekov. On a class of problems of determining the temperature and density of heat sources given initial and final temperature // Siber. Math. J. 53:1, 146-151 (2012).

22. W. Rundell. Determination of an unknown nonhomogeneous term in a linear partial differential equation from overspecified boundary data // Appl. Anal. 10:3, 231-242 (1980).

23. M. Ruzhansky, N. Tokmagambetov, B. T. Torebek. Inverse source problems for positive operators. I: Hypoelliptic diffusion and subdiffusion equations // J. Inverse Ill-posed Probl. 27:6, 891-911 (2019).

24. W. Rundell, Z. Zhang. Recovering an unknown source in a fractional diffusion problem // J. Comput. Phys. 368, 299-314 (2018).

25. T. Simon. Comparing Frechet and positive stable laws // Electron. J. Probab. 19:16, 1-25 (2014).

26. M. M. Slodička, K. Šškova, K. V. Bockstal. Uniqueness for an inverse source problem of determining a space dependent source in a time-fractional diffusion equation // Appl. Math. Lett. 91, 15-21 (2019).

27. D. Tong, Y. Liu. Exact solutions for the unsteady rotational flow of non-Newtonian fluid in an annular pipe // Inter. J. Engin. Sci. 43:3-4, 281-289 (2005).

28. B.T. Torebek, R. Tapdigoglu. Some inverse problems for the nonlocal heat equation with Caputo fractional derivative // Math. Methods Appl. Sci. 40:18, 6468-6479 (2017).

Daurenbek Serikbaev,

Al-Farabi Kazakh National University,

Al-Farabi av. 71,

050040, Almaty, Kazakhstan

Department of Mathematics: Analysis, Logic and Discrete Mathematics,

Ghent University,

Krijgslaan 281,

B-9000, Gent, Belgium

Institute of Mathematics and Mathematical Modeling,

Pushkin str., 125,

Almaty, Kazakhstan

E-mail: daurenbek.serikbaev@ugent.be 